\title{
AVALIAÇÃO INTRA-OPERATÓRIA DA PRESSÃO PORTAL E RESULTADOS IMEDIATOS DO TRATAMENTO CIRÚRGICO DA HIPERTENSÃO PORTAL EM PACIENTES ESQUISTOSSOMÓTICOS SUBMETIDOS A DESCONEXÃO ÁZIGO-PORTAL E ESPLENECTOMIA
}

\author{
Walter De Biase da SILVA-NETO ${ }^{1}$, Adalberto CAVARZAN ${ }^{1}$ e Paulo HERMAN ${ }^{2}$
}

RESUMO - Racional - No Brasil a principal causa de hipertensão portal é a esquistossomose mansônica na sua forma hepatoesplênica. Com relação ao seu tratamento, a preferência da maioria dos autores no Brasil recai sobre a desconexão ázigo-portal e esplenectomia geralmente associada à escleroterapia endoscópica pós-operatória para tratamento dessa enfermidade. No entanto, não estão bem estabelecidas as alterações hemodinâmicas portais decorrentes do tratamento cirúrgico da hipertensão portal e sua influência no resultado desse tratamento. Objetivos - Avaliar o impacto imediato da desconexão ázigo-portal e esplenectomia na pressão portal e também os resultados do tratamento cirúrgico da hipertensão portal no que se refere à recidiva hemorrágica e ao calibre das varizes de esôfago. Casuística e Método - Foram estudados 19 pacientes com esquistossomose hepatoesplênica e hipertensão portal, com história de hemorragia digestiva alta por ruptura de varizes esofágicas, com idade média de 37,9 anos. Estes pacientes não haviam sido submetidos a tratamento prévio e foram, eletivamente, tratados cirurgicamente com desconexão ázigo-portal e esplenectomia. Durante a cirurgia, foi avaliada a pressão portal, no início e no final do procedimento, através da cateterização da veia porta por cateter de polietileno introduzido por veia jejunal. Todos os pacientes foram submetidos a endoscopia no pré e no pós-operatório (em torno do $60^{\circ}$ dia do pós-operatório) para avaliar, segundo classificação de Palmer, a variação do calibre das varizes esofagianas após a desconexão ázigo-portal e esplenectomia. Resultados - Todos os pacientes apresentaram queda da pressão portal, sendo a média desta queda, após a desconexão ázigo-portal e esplenectomia, de 31,3\%. Na avaliação pós-operatória (endoscopia após cerca de 60 dias) houve redução significativa do calibre das varizes esofagianas quando comparadas ao pré-operatório. Conclusão - A desconexão ázigo-portal e esplenectomia promoveram queda imediata na pressão portal, com conseqüente diminuição do calibre das varizes esofágicas. Observou-se ainda que não é insignificante o risco de mortalidade e complicações graves relacionados a essa técnica.

DESCRITORES - Hipertensão portal, cirurgia. Pressão na veia porta. Esplenectomia.

\section{INTRODUÇÃO}

A esquistossomose mansônica se apresenta no Brasil como doença endêmica de alta prevalência, acometendo cerca de 12 milhões de pessoas ${ }^{(12,23,29)}$. Sua forma hepatoesplênica atinge cerca de 1 milhão de pessoas, tornando-a a maior causa de hipertensão portal em nosso meio.
Dentre os tratamentos existentes para a hipertensão portal esquistossomótica, o clínico tem eficácia duvidosa, uma vez que não alcança o objetivo principal: eliminação ou, pelo menos, redução do calibre das varizes esofagianas, impedindo assim, o ressangramento ${ }^{(37)}$. O tratamento endoscópico, apresenta índices elevados de ressangramento quando utilizado isoladamente, entre $11 \%$ e $33,3 \%^{(3,4,11,14,16,24,34)}$.

Trabalho realizado com a associação dos Departamentos de 'Cirurgia da Faculdade de Medicina da Universidade Federal de Goiás, Goiânia, GO e de ${ }^{2}$ Gastroenterologia da Faculdade de Medicina da Universidade de São Paulo, SP.

Endereço para correspondência: Dr. Walter De Biase da Silva Neto - Av. Ismerino S. de Carvalho, 524 - St. Aeroporto - 74075-040 - Goiânia, GO. 
O tratamento cirúrgico é a terapêutica de escolha na maioria dos Serviços que lidam com esta doença, no entanto, duas opções dividem a indicação dos autores: as cirurgias de derivação portal seletiva, notadamente a cirurgia de derivação esplenorrenal seletiva ${ }^{(3)}$, que objetiva a descompressão seletiva do território portal esquerdo, sítio das varizes esofagianas, e as desconexões ázigo-portais, que visam interromper a circulação colateral do sistema portal em direção ao esôfago, onde há varizes ${ }^{(6)}$.

As cirurgias de derivação portal seletiva são efetivas no controle do ressangramento, porém são acompanhadas de índices consideráveis de encefalopatia portossistêmica pós-operatória - entre 3,3\% e $14,8 \%^{(1,2,}$ $14,17,18,27,31,33)$ - o que torna sua indicação em um paciente com função hepática normal pouco aconselhável.

As cirurgias de desconexão ázigo-portal, no entanto, apresentam índice de ressangramento significativo entre $6 \%$ e $29 \%$, porém sem o inconveniente da encefalopatia portossistêmica ${ }^{(9,15,17,19,20,22,31)}$. Estudos realizados na década de 90, como os de SAKAI et al. ${ }^{(34)}$ e PUGLIESI ${ }^{(30)}$, mostraram queda significativa no índice de ressangramento, quando se associou à cirurgia a escleroterapia endoscópica no pós-operatório. Estes resultados fazem com que a desconexão ázigo-portal e esplenectomia (DAPE) tenha a preferência da maioria dos autores como o tratamento de escolha em pacientes esquistossomóticos com varizes esofagianas sangrantes ${ }^{(5,6,7,10,20,30)}$.

Em nosso meio existem poucos estudos sobre a hemodinâmica portal em esquistossomóticos submetidos a DAPE e, ainda assim, seus resultados são controversos. CAPUA Jr. et al. ${ }^{\left({ }^{()}\right.}$e STELMACH e CAPUA Jr. ${ }^{(36)}$ não encontraram alterações significativas na pressão portal após a DAPE. Já CLEVA ${ }^{(10)}$ observou queda, em média, de cerca de $30 \%$ após o mesmo procedimento. Ainda POLLARA ${ }^{(28)}$, em 1992, também utilizando a desconexão ázigo-portal porém realizando apenas a ligadura da artéria esplênica, sem a esplenectomia, encontrou queda média de cerca de $25 \%$ da pressão portal após o procedimento.

Em virtude dessas controvérsias e no intuito de obter subsídios que contribuam para a escolha do melhor tratamento para pacientes com esquistossomose com varizes esofagianas sangrantes, foi objetivo deste estudo avaliar o impacto intra-operatório da DAPE sobre a pressão portal e os resultados imediatos do tratamento cirúrgico da hipertensão portal por esquistossomose, forma hepatoesplênica, tratados por DAPE.

\section{CASUÍSTICA}

Foram estudados retrospectivamente 19 pacientes com hipertensão portal decorrente de esquistossomose hepatoesplênica, sendo 11 do sexo masculino e 8 do feminino, com idade média de 37,9 anos (variando entre 18 e 61 anos), história de hemorragia digestiva alta por ruptura de varizes de esôfago. Nenhum paciente havia sido submetido a tratamento (clínico ou endoscópico) prévio. Todos foram submetidos a tratamento cirúrgico de forma eletiva (DAPE), sendo 17 operados no Departamento de Clínica Cirúrgica da Faculdade de Medicina da Universidade Federal de Goiás e 2 no Serviço de Cirurgia Geral do Hospital Geral de Goiânia, pela mesma equipe, no período de janeiro de 1992 a março de 2001.

\section{Critérios de inclusão}

- Diagnóstico de esquistossomose mansônica baseado em evidências epidemiológicas, clínicas e confirmados por exame histopatológico;

- presença de antecedente de hemorragia digestiva alta por rotura de varizes de esôfago.

\section{Critérios de exclusão}

- História de etilismo crônico;

- evidência clínica ou laboratorial de insuficiência hepatocelular;

- sorologia positiva para hepatite B e/ou C;

- evidência de outras hepatopatias ao exame histopatológico.

Todos os pacientes foram submetidos a avaliação laboratorial da função hepática e a exame endoscópico no período pré-operatório.

As varizes esofagianas foram avaliadas por endoscopia e classificadas de acordo com o seu diâmetro (segundo classificação de PALMER e BRICKS $^{(26)}$ ):

grau $\mathrm{I}=$ varizes com diâmetro inferior a $3 \mathrm{~mm}$

grau II $=$ varizes com diâmetro entre 3 e $6 \mathrm{~mm}$

grau III = varizes com diâmetro superior a $6 \mathrm{~mm}$

A cirurgia era realizada eletivamente com o paciente sob anestesia geral em decúbito dorsal horizontal, com monitorização intra-operatória da freqüência cardíaca, pressão venosa central e pressão arterial média invasiva. A técnica operatória consistiu de laparotomia mediana desde o apêndice xifóide até a cicatriz umbilical; biopsia hepática em cunha do lobo direito, para estudo histopatológico; cateterização de ramo venoso mesentérico (do mesojejunal) com cateter de polietileno de 6 Fr e progressão deste no sentido da veia porta, por uma extensão de cerca de $15 \mathrm{~cm}$, sendo sua localização confirmada pela palpação do cateter no interior da veia porta. $\mathrm{O}$ cateter era conectado a um sistema de coluna de água graduado em centímetros, estando a marca inicial ao nível da linha axilar média; aferição da pressão portal inicial concomitante a da pressão arterial, freqüência cardíaca e pressão venosa central. Ligadura prévia da artéria esplênica ao nível da transição corpo-caudal do pâncreas com posterior esplenectomia. Procediase, então, à desvascularização do terço inferior do esôfago, fundo e corpo gástrico, vasos breves, preservando os nervos vagos, seguida de ligadura dos vasos da pequena curvatura desde a incisura angular, até a extensão de 5 a $7 \mathrm{~cm}$ no sentido proximal no esôfago. Realizava-se, então, uma gastrofundoplicatura póstero-lateral ( $2 / 3$ por uma extensão longitudinal de $5 \mathrm{~cm}$ ). Ao final do procedimento, aferição da pressão portal concomitante a da pressão arterial, freqüência cardíaca e pressão venosa central com posterior retirada do cateter mesentérico-portal. Fechamento da cavidade por planos.

Foram analisados:

A - Parâmetros hemodinâmicos (sistêmicos e portais) intraoperatórios

1- pressão arterial média

2- freqüência cardíaca

3- pressão venosa central

4- pressão portal

B - Calibre das varizes esofagianas

1- pré-operatório

2- pós-operatório precoce (60 dias)

A análise dos resultados obtidos foi realizada através de testes estatísticos em que, para variáveis quantitativas, foi usado o teste paramétrico $t$ de Student para dados pareados, que se referem ao mesmo grupo de pacientes, com informações em períodos distintos, e o não-paramétrico de Wilcoxon para dados qualitativos no decorrer do tempo.

Após a alta hospitalar, os pacientes eram acompanhados em ambulatório e submetidos a avaliação endoscópica cerca de 60 dias após a cirurgia (a necessidade de escleroterapia era determinada pelo endoscopista). 
Os resultados das endoscopias foram analisados no período pósoperatório (cerca de 30 dias) e a análise das varizes consistiu em se avaliar se elas desapareciam, diminuíam, aumentavam ou permaneciam inalteradas.

\section{RESULTADOS}

Todos os pacientes apresentavam provas de função hepática dentro dos limites da normalidade.

Tanto a pressão arterial, quanto a freqüência cardíaca e a pressão venosa central aferidas, concomitantemente às medidas do início e do fim da operação, não apresentaram variação significativa.

Todos os pacientes apresentaram queda da pressão portal, quando se comparou o início e o final do procedimento: $10,5 \%$ dos pacientes com queda $<10 \%, 26,3 \%$ apresentaram queda $>40 \%$ (Tabela 1 ). A pressão portal média apresentou queda significativa de $31,3 \mathrm{~cm}$ de $\mathrm{H}_{2} \mathrm{O}$, para um valor médio de $22 \mathrm{~cm}$ de $\mathrm{H}_{2} \mathrm{O}(31,3 \%)$, conforme observado na Tabela 2.

O tempo operatório médio foi de 282,1 minutos, variando de 255 a 360 minutos.

TABELA 1 - Avaliação da pressão portal intra-operatória em 19 pacientes submetidos a desconexão ázigo-portal e esplenectomia

\begin{tabular}{lccc}
\hline Caso & Pp inicial & Pp final & \% de queda \\
\hline 1 & 26 & 15 & 42,3 \\
2 & 37 & 27 & 27,1 \\
3 & 31 & 29 & 6,5 \\
4 & 37 & 22 & 40,6 \\
5 & 24 & 19 & 20,9 \\
6 & 29 & 19 & 34,5 \\
7 & 36 & 23 & 36,1 \\
8 & 23 & 21 & 8,7 \\
9 & 36 & 24 & 33,4 \\
10 & 38 & 24 & 36,9 \\
11 & 39 & 19 & 51,3 \\
12 & 29 & 20 & 31,1 \\
13 & 30 & 20 & 33,4 \\
14 & 30 & 24 & 20,0 \\
15 & 38 & 27 & 29,0 \\
16 & 34 & 26 & 23,6 \\
17 & 29 & 22 & 24,2 \\
18 & 38 & 22 & 42,1 \\
19 & 41 & 21 & 48,8 \\
\hline Pp= pressão portal & & &
\end{tabular}

TABELA 2 - Pressão portal média no início e ao final da desconexão ázigo-portal e esplenectomia

\begin{tabular}{lcc}
\hline & \multicolumn{2}{c}{ Média } \\
\cline { 2 - 3 } Pressão portal $(\mathrm{cmH} 2 \mathrm{O})$ & Inicial & Final \\
& $33,00 \pm 5,28$ & $22,32 \pm 3,43$ \\
\hline$P=0,0001$ &
\end{tabular}

As complicações decorrentes do ato operatório ocorreram em 36,8\% dos pacientes e foram: íleo adinâmico em cinco pacientes $(26,3 \%)$, isquemia mesentérica em dois pacientes $(10,5 \%)$, trombose parcial da veia porta em um paciente $(5,2 \%)$ e trombose total em um, infecção do trato urinário em um paciente $(5,2 \%)$, abscesso subfrênico em um paciente $(5,2 \%)$ e um caso de necrose de fundo gástrico $(5,2 \%)$. Não se observaram complicações relacionadas à colocação do cateter na veia porta.

Três pacientes $(15,7 \%)$ evoluíram a óbito: um por septicemia secundária à pneumonia no $60^{\circ}$ pós-operatório, um por coagulação intravascular disseminada (CIVD), secundária a um grande coágulo intra-peritonial no $5^{\circ}$ pós-operatório, e outro por septicemia pósperfuração por necrose isquêmica da transição corpo/fundo gástrico no $13^{\circ}$ pós-operatório.

Quando se comparou a variação do calibre das varizes esofagianas no pré e no pós-operatório (60 dias após a DAPE), observou-se redução significativa deste, como pode ser observado na Tabela 3.

TABELA 3 - Calibre das varizes esofagianas nos períodos pré e pósoperatório (60 dias)

\begin{tabular}{lcccc}
\hline \multirow{2}{*}{$\begin{array}{l}\text { Classificação das varizes } \\
\text { segundo Palmer }\end{array}$} & \multicolumn{2}{c}{ Pré-operatório } & \multicolumn{2}{c}{ Pós-operatório } \\
\cline { 2 - 5 } & Número & $\%$ & Número & $(\%)$ \\
\hline 0 & - & 0,0 & - & 0,0 \\
I & 1 & 5,3 & 6 & 42,9 \\
II & 2 & 10,5 & 6 & 42,9 \\
III & 16 & 84,2 & 2 & 14,3 \\
Total & 19 & 100,0 & 14 & 100,0 \\
\hline$P=0,004$ & & & &
\end{tabular}

\section{DISCUSSÃO}

Pelo fato de ser doença de alta prevalência em nossa população e com potencial complicação grave em sua forma hepatoesplênica, a esquistossomose representa doença de forte impacto social.

Assim, a necessidade de tratamento eficiente que controle efetivamente a hemorragia digestiva com poucos efeitos colaterais, se torna evidente. Neste sentido, a preferência dos autores recai sobre o tratamento cirúrgico e, na maioria dos Serviços que tratam desta afecção, a cirurgia de eleição é a $\operatorname{DAPE}^{(5,6,7,10,20,30)}$. Para diminuir os índices de ressangramento, maior inconveniente da DAPE, associa-se a esta um tratamento complementar, como a escleroterapia endoscópica pós-operatória, com bons resultados ${ }^{(5,30,34,35)}$. Este bom resultado em relação à taxa de ressangramento pós-operatório, também foi observado no presente estudo, uma vez que apenas um paciente $(8,3 \%)$ apresentou hemorragia digestiva pós-operatória, sendo esta decorrente de sangramento em úlcera gástrica.

Em relação aos efeitos da DAPE sobre a hemodinâmica portal, os resultados são controversos. Enquanto CLEVA $^{(10)}$ e POLLARA ${ }^{(28)}$ encontraram queda de $30 \%$ e $25 \%$, respectivamente, da pressão portal, logo após a ligadura da artéria esplênica, CAPUA et al. ${ }^{(6)} \mathrm{e}$ STELMACH e CAPUA ${ }^{(36)}$, avaliando a pressão portal intra-operatória encontraram, ao final da DAPE, valores próximos dos iniciais. Na presente série, todos os pacientes apresentaram queda na pressão portal e a diferença entre a pressão portal média no início e ao final da DAPE foi de 31,3\%.

A provável causa da queda na pressão portal após a DAPE é a retirada da circulação esplênica do circuito sistêmico-portal como resultado da esplenectomia, como observado por $\operatorname{CLEVA}^{(10)}$ que, a partir da ligadura da artéria esplênica, haveria interrupção desta circulação hiperdinâmica, com conseqüente queda na pressão portal. Esta assertiva é corroborada neste mesmo estudo onde se demonstrou queda de $28 \%$ do fluxo portal após a ligadura da artéria esplênica; além disto, também se observou que tanto o fluxo, quanto a pressão portal mantiveram-se estáveis após a esplenectomia e desvascularização esôfago-gástrica. POLLARA ${ }^{(28)}$ também havia demonstrado queda da pressão portal de cerca de $25 \%$ após a ligadura da artéria esplênica. Esta queda da pressão portal pode ser um dos fatores para explicar os bons resultados da DAPE no tratamento de pacientes com hipertensão portal por esquistossomose. MOSSIMANN et al. ${ }^{(25)}$ demonstraram que a queda da pressão portal 
tem relação direta com a queda da pressão nas varizes esofágicas.

Neste estudo houve diminuição significativa do calibre das varizes esofagianas, quando comparados o pré e pós-operatório imediato (60 dias), observação semelhante à de outros estudos ${ }^{(9,32,38)}$. Embora na presente casuística não se tenha observado o desaparecimento das varizes, como descrito em alguns casos por outros autores ${ }^{(18,30)}$, a diminuição do calibre das varizes esofagianas encontradas após a DAPE além de facilitar a escleroterapia endoscópica ${ }^{(34)}$, também diminui a possibilidade de ressangramento e, se este ocorrer, terá menor intensidade e gravidade do que em pacientes com varizes de grosso calibre, como demonstrou CURY ${ }^{(13)}$, em 1989. Esse autor acompanhou pacientes esquistossomóticos e encontrou correlação direta entre o maior calibre das varizes esofagianas e maior freqüência e gravidade dos episódios de sangramento.

Três pacientes $(15,7 \%)$ apresentaram, no pós-operatório, quadro de dor abdominal de moderada intensidade, distensão abdominal, febre e, em dois destes, confirmou-se a suspeita clínica de trombose da veia porta após a realização de ultra-som Doppler. A incidência de trombose de veia porta na literatura varia entre $13,2 \%$ e $53,2 \%{ }^{(19,29)}$ e é considerada a complicação pós-operatória mais freqüente da DAPE. Esta complicação tem como causa provável a diminuição do fluxo sangüíneo portal, após a esplenectomia associada à estagnação do sangue portal, causada pela ligadura das veias colaterais ${ }^{(10)}$ e também pelo aumento de plaquetas que se segue à esplenectomia nesses pacientes ${ }^{(8)}$.

A isquemia mesentérica ocorreu em dois casos (10\%), uma delas acometeu pequeno segmento jejunal e evoluiu favoravelmente após ressecção intestinal. Já em outro caso, a isquemia estava associada à trombose da veia porta e a paciente evoluiu desfavoravelmente com fístula, peritonite, septicemia e óbito. A trombose mesentérica após a DAPE também foi demonstrada por CHAIB et al. ${ }^{\left({ }^{(8)}\right.}$, em relato de três casos, em que dois evoluíram bem com medidas de suporte clínico e um necessitou de seguidos procedimentos de ressecção intestinal, culminando com o óbito. Os mecanismos que levariam a essa trombose ainda não estão bem esclarecidos, porém, como já citados, dois fatores predisponentes à trombose venosa estão presentes: a estase venosa e a trombocitose, que acontecem após a retirada do baço e podem ser lembrados como possíveis precipitadores tanto para a trombose ao nível da veia porta, como sua extensão para o tronco mesentérico.

A mortalidade operatória ocorreu em 15,7\% dos pacientes (três casos): um por CIVD, secundária à coagulopatia de consumo por coágulos intra-abdominais, um por peritonite e septicemia, desencadeada por necrose do fundo gástrico decorrente de isquemia no sítio de ligadura de vaso durante a "esqueletização" da grande curvatura, e outro por septicemia secundária à fístula após ressecção intestinal. A presente série apresenta elevado índice de óbitos, porém é interessante destacar que estes ocorreram no início do estudo, período em que ainda era pequena a experiência do Serviço com o procedimento, além da maior precariedade de condições de acompanhamento pós-operatório desses pacientes. O número de óbitos encontrado em uma amostra pequena (19 casos) cria a falsa impressão de que esse procedimento apresenta elevado índice de mortalidade. Índices variáveis de mortalidade foram encontrados em alguns estudos, entre $5,3 \%$ e $13,6 \%{ }^{(5,20,21)}$. No entanto, em outros estudos, não foram observados óbitos na casuística ${ }^{(10,28,30)}$. Ainda assim deve-se ressaltar que, embora a DAPE seja cirurgia de relativa facilidade técnica, está associada a complicações potencialmente letais e essa possibilidade não deve ser desprezada durante a programação do ato operatório, ficando patente que esse procedimento deve ser realizado em centros médicos especializados com cirurgiões experientes e com suporte técnico intra e pós-operatório adequado.

Demonstrou-se que a DAPE diminui a pressão portal, já no intraoperatório e que muito provavelmente, como conseqüência, ocorre diminuição significativa no calibre das varizes esofágicas. Esses fatores devem ser importantes para os bons resultados no tratamento da hipertensão portal na esquistossomose hepatoesplênica. Salienta-se, porém, que essa operação é passível de complicações graves e apresenta risco significativo de mortalidade.

Silva-Neto WDB, Cavarzan A, Herman P. Intra-operative evaluation of portal pressure and immediate results of surgical treatment of portal hypertension in schistosomotic patients submitted to esophagogastric devascularization with splenectomy. Arq Gastroenterol 2004;41(3):150-4.

ABSTRACT - Background - The main cause of portal hypertension in Brazil is the hepato-splenic form of mansonic schistosomiasis and the most employed technique for the surgical approach of this disease is the esophagogastric devascularization with splenectomy, generally associated to postoperative endoscopical esophageal varices sclerotherapy. The hemodynamic alterations after surgical treatment and its possible influence on the outcome are not well established. Aim - To evaluate the immediate impact of esophagogastric devascularization with splenectomy upon portal pressure as well as the results of the surgical treatment on digestive hemorrhage recurrence and on esophageal varices. Methods - Nineteen patients with mean age of 37.9 years and portal hypertension and previous episodes of digestive hemorrhage caused by esophageal varices rupture due to hepato-splenic schistosomiasis were studied. None of the patients had received any treatment prior to the surgery and underwent to elective esophagogastric devascularization with splenectomy. Portal pressure was assessed at the beginning and at the end of esophagogastric devascularization with splenectomy through portal vein catheterization with a polyethylene catheter introduced through a jejunal branch vein. All patients were submitted to digestive endoscopy before and after the surgery, in order to classify the size of esophageal varices after esophagogastric devascularization with splenectomy according to Palmer's classification. Results - In all patients the portal pressure had diminished with a mean decrease of $31.3 \%$ after esophagogastric devascularization with splenectomy. In the postoperative endoscopic follow-up (1 month), the esophageal varices showed a statistically significant reduction in their size, when compared to the pre-surgical measurements. Conclusion - These results have demonstrated that the esophagogastric devascularization with splenectomy promotes immediate decrease in the portal pressure and a consequent reduction in the esophageal varices size. We also observed that the risk of mortality and severe complications related to this technique is not insignificant.

HEADINGS - Portal hypertension, surgery. Portal pressure. Splenectomy. 


\section{REFERÊNCIAS BIBLIOGRÁFICAS}

1. Abrantes W L. Hipertensão portal. In: Dani R, Castro LP, editores. Gastroenterologia clínica. Rio de Janeiro: Guanabara Koogan; 1988. p.983-1000.

2. Abrantes WL. As derivações venosas no tratamento das varizes do esôfago. In: Leonardi LS, editor. Controvérsias na cirurgia do aparelho digestivo. Rio de Janeiro: Medsi; 1991. p.283-92

3. Al Karawi MA, Mohamed AE, Hanid MA, Al-Otaibi R, Shamed M. Favourable outcome in sclerotherapy for bleeding oesophageal varices in schistosomiasis: results in 30 patients. J Gastroenterol Hepatol 1986;1:151-7.

4. Al Karawi MA, Mohamed ARES, Ahmed AMM, Shariq S, Yasawy MI. Long-term outcome of endoscopic sclerotherapy of variceal bleeding: comparative study between schistosomiasis and others. Hepatogastroenterology 1996;43:287-91.

5. Boin IFSF. Desvascularização esofagogástrica ampla associada à esplenectomia e escleroterapia programada no pós-operatório no tratamento das varizes esofagogástricas na esquistossomose hepatoesplênica [dissertação]. Campinas, Faculdade de Ciências Médicas da Universidade Estadual de Campinas; 1991

6. Capua Jr A, Szutan LA, Saad Jr R, Stelmach D, Assef JC. Alterações da pressão porta de doentes esquistossomóticos submetidos a operação de esplenectomia e desconexão ázigo-portal. GED Gastroenterol Endosc Dig 1992;11:1.

7. Carneiro JLA. A circulação colateral gastresofágica após desconexão ázigo-portal. Portografia trans-hepática na esquistossomose mansônica [tese livre-docência]. Vitória: Centro Biomédico da Universidade Federal do Espírito Santo; 1979.

8. Chaib E, Herman P, D'Albuquerque LC, Pugliesi V, Antônio LGM, Feijó LFA, Ishida RY, Saad WA, Pinotti HW. Trombose mesentérica após a desconexão ázigo-portal com esplenectomia para o tratamento das varizes esofagianas sangrantes na esquistossomose mansônica. Relato de três casos. Rev Hosp Clín Fac Med S Paulo 1996;51:65-8.

9. Chaib SA, Lessa BS, Cecconello I, Felix VN, Chaib E. A new procedure for the treatment of bleeding esophageal varices by transgastric azigo-portal disconnection. Int Surg 1983;68:353-6.

10. Cleva R. Hemodinâmica sistêmica e portal em doentes esquistossomóticos, forma hepatoesplênica, submetidos à desconexão ázigo-portal, com esplenectomia (DAPE) [tese]. São Paulo: Faculdade de Medicina da Universidade de São Paulo; 1996.

11. Cordeiro F. Variceal sclerosis in schistosomotic patients: a five-year follow-up study. Gastrointest Endosc 1990;36:475-8.

12. Coutinho AD, Domingues ALC. Esquistossomose mansoni. In: Dani R, Castro LP, editores Gastroenterologia clínica. Rio de Janeiro: Guanabara Koogan; 1988. p.1361-86.

13. Cury AA. Hepato-splenic form of the schistosomiasis: contributions to the natural history. Rev Col Bras Cir 1989;16:117-21.

14. Cury AA. Anastomose espleno-renal distal seletiva versus esclerose endoscópica para o controle das varizes esofagianas em pacientes portadores de esquistossomose. Rev Col Bras Cir 1990;17:106-9.

15. El Masri SH, Hassan MA. Splenectomy and vasoligation for patients with haematemesis secondary to bilharzial hepatic fibrosis. Br J Surg 1982;69:314-6.

16. El Zayadi A, El-Din SS, Kabil SM. Endoscopic scleroterapy versus medical treatment for bleeding esophageal varices in patients with schistosomal liver disease. Gastrointes Endosc 1988;34:314-7.

17. Ezzat FA, Abu-Elmagd KM, Aly IY, Aly MA, Fathy OM, El-Barbary MH, Bahgat OO, Salam AA, Kutner MH. Distal splenorenal shunt for management of variceal bleeding in patients with schistosomal hepatic fibrosis. Ann Surg 1986;204:566-73.

18. Ferraz AAB, Lopes EPA, Bacelar TS, Silveira MJC, Silva LMM, Ferraz EM. Tratamento cirúrgico da hipertensão portal esquistossomótica no HC/UFPE - Análise de 131 casos. Rev Col Bras Cir 1999;27:5.

19. FerrazAAB. Tratamento cirúrgico da hipertensão porta esquistossomótica na UFPE: aspectos históricos e contemporâneos. An Fac Med Univ Fed Pernambuco 2000;45:153-4.

20. Ferraz AAB, Bacelar TS, Marcello JCS, Coelho ARB, Câmara-Neto RD, Araújo Jr JGC, Ferraz E M. Surgical treatment of schistosomal portal hypertension. Int Surg $2001 ; 86: 1-8$
21. Haddad CM, Pan Chacon J, Herani-Filho B, Kobata C. Desvascularização gastroesofágica e esplenectomia no tratamento de varizes esôfago-gástricas. Rev Col Bras Cir 1981;8:6

22. Kelner S, Ferreira PR, Dantas A, Lima-Filho JFC, Souza AP, Carreiro Jr JCP, Ferraz EM, Silveira M, Coêlho ARB, Câmara-Neto RD, Domingues LAW. Ligadura de varizes esôfago-gástricas na hipertensão porta esquistossomótica: avaliação de 25 anos. Rev Col Bras Cir 1982;9:140-6.

23. Machado PA. Programa especial de controle da esquistossomose: o modelo. In: $6^{a}$ Conferência Nacional de Saúde. Brasília, DF, Ministério da Saúde, 1977; p.267-84.

24. Mohamed AE, Al-Karawi MA, Al-Otaibi R, Hanid MA. Results of sclerotherapy in 100 patients: comparison of the outcome between schistosomiasis and hepatitis B. Hepatogastroenterology 1989;36:333-6.

25. Mossimann R, Bohnet J, Gartsch Ph. Endoscopic non-agressive assessment of oesophageal variceal pressure compared with wedged hepatic venous pressure in alcoholic liver cirrhosis. Endoscopy 1983;15:101-3.

26. Palmer ED, Bricks IB. Correlation between the severity of esophageal varices in cirrhosis and their propensity toward hemorrage. Gastroenterology 1956;30:85-90.

27. Pitanga LC. Selective splenorenal anastomosis technical details and results of 340 patients subjected in 15 years. Dig Dis Sci 1986;31:398S.

28. Pollara WM. Desvascularização esôfago-gástrica por via transmediastinal sem esplenectomia no tratamento das varizes do esôfago. Avaliação clínica [tese livre docência]. São Paulo: Faculdade de Medicina da Universidade de São Paulo; 1992.

29. Prata A. Infection with $S$. mansoni. In: Jordan P, Webbe G, editors. Schistosomiasis: epidemiology, treatment a control. London: William Heinemann; 1982. p.105-27.

30. Pugliesi V. Desconexão ázigo-portal e esplenectomia associados à escleroterapia endoscópica no tratamento das varizes do esôfago na esquistossomose hepato-esplênica: avaliação de parâmetros clínicos, laboratoriais e hemodinâmicos portais [tese]. São Paulo: Faculdade de Medicina da Universidade de São Paulo; 1996.

31. Raia S, Silva LC, Gayotto LCC, Forster SC, Fukushima J, Strauss E. Portal hypertension in schistossomiasis: a long-term follow-up of a randomized trial comparing three types of surgery. Hepatology 1994;20:398-403.

32. Rocha JRM, Machado MAC, Bove CR, Tokikaka GH, Machado MCC. Contribuição ao estudo da desvascularização da transição esofagogástrica no tratamento das varizes sangrantes do esôfago. Rev Hosp Clín Fac Med S Paulo 1998;53:249-53.

33. Saad WA, Yamin H, Freday U, Velard G, Chaib S, Parra OM, Berman A, Vasconcelos E. Descompressão seletiva do sistema portal no tratamento da hipertensão portal associada a varizes do esôfago sangrantes. AMB Rev Assoc Med Bras 1977;23:165-8.

34. Sakai P, Boaventura S, Ishioka S, Mies S, Sette H, Pinotti HW. Sclerotherapy of bleeding esophageal varices in shistosomiasis - comparative study in patients with and without previous surgery for portal hypertention. Endoscopy 1990;22:5-7.

35. Sakai P. Esclerose endoscópica das varizes esofágicas após tratamento cirúrgico da hipertensão portal em pacientes com esquistossomose hepatoesplênica. Arq Gastroenterol 2001;38:2

36. Stelmach D, Cápua Jr A. Estudo da pressão portal no pós-operatório de doentes esquistossomóticos submetidos a esplenectomia e desvascularização gastro-esofágica. GED Gastroenterol Endosc Dig 1996:5:41-8.

37. Strauss E. Tratamento medicamentoso da hipertensão portal: realidade ou esperança? Rev Bras Clin Ter 1983;12:275-6.

38. Strauss E, Sakai P, Gayotto LCC, Cardoso RA, Forster S, Raia S. Size of gastroesophageal varices: its behavior after the surgical treatment of porta hypertension. Rev Hosp Clín Fac Med S Paulo 1999;54:193-8. 